\title{
LA PROCEDENCIA CUANTITATIVA Y CUALITATI- VA DE LOS ENCUADRES SOCIALES: EL CASO DE LAS ELECCIONES GENERALES ESPAÑOLAS DE 2008 Y 2011
}

THE QUANTITATIVE AND QUALITATIVE ORIGIN OF THE SOCIAL FRAMES: THE CASE OF THE SPANISH GENERAL ELECTIONS OF 2008 AND $2011^{1}$

\author{
Antón R. Castromil \\ Universidad Complutense de Madrid \\ arcastromil@ccinf.ucm.es \\ Palmira Chavero \\ FLACSO \\ pchavero@flacso.edu.ec
}

\section{Resumen}

Este artículo, como el monográfico de la revista en la que se inserta, sirve de homenaje al profesor Fermín Bouza Álvarez, recientemente fallecido. Maestro, cómplice, confidente y amigo, Fermín influyó de manera decisiva en un buen número de investigadores entre los que tenemos la suerte de encontramos. Además de los autores de esta contribución, es justo que mencionar a Juan Jesús González y Raquel Rodríguez. En torno a la figura de Fermín formamos el conocido como equipo "Agenda y voto". El legado de Fermín sigue vivo en todos nosotros. El artículo que presentamos a continuación pretende dar muestra los principales trabajos del equipo "Agenda y voto". De ahí la importante carga de auto citas y referencias a proyectos de investigación capitaneados tanto por Fermín como por el profesor Juan Jesús González. Quizá el mayor tesoro que Fermín nos ha legado haya sido el uso de dos enfoques complementarios que

\footnotetext{
${ }^{1}$ Una versión preliminar en inglés de este artículo fue defendida en el XXIII Congreso Mundial de Ciencia Política celebrado en Montreal, Canadá, en el verano de 2014..
} 
resultan idóneos para la toma en consideración de los efectos de los medios de comunicación en la ciudadanía de los sistemas democráticos actuales. Nos referimos al empleo de la teoría de agenda setting y a la aplicación a los estudios de comunicación de la teoría del encuadre o framing. Este trabajo pretende arrojar un poco más de luz sobre la última de estas dos perspectivas, desgranando dos tipos de encuadre con procedencias bien distintas: La "vía cuantitativa o demoscópica" y la "vía cualitativa o mediática" del framing. La primera de ellas es posible construirla a través de encuestas y la segunda mediante análisis de contenido de medios de comunicación. Como defenderemos en las páginas que siguen, se trata de dos vías complementarias que ofrecen, trabajando juntas, una perspectiva mucho más completa del funcionamiento de los encuadres en la sociedad.

Palabras clave: Framing, frame, medios de comunicación, encuestas, campaña electoral, España

\section{Abstract}

This article, like the monograph of the journal in which it is published, serves as a tribute to Professor Fermín Bouza Álvarez, recently deceased. Master, accomplice, confidant and friend, Fermín decisively influenced a good number of researchers among whom we were fortunate to be. In addition to the authors of this contribution, it is fair to mention Juan Jesús González and Raquel Rodríguez. Around the figure of Fermín we formed the one known as the "Agenda y voto" team. Fermin's legacy is still alive in all of us. The article presented below aims to show the main work of the "Agenda and voting" team. Hence the important burden of self-citations and references to research projects led by both Fermín and Professor Juan Jesús González. Perhaps the greatest treasure that Fermín has bequeathed us has been the use of two complementary approaches that are ideal to consider the effects of the media on the citizenship of the current democratic systems. We refer to the use of agenda setting theory and the application to communication studies of framing theory. This work aims to shed more light on the latter of these two perspectives, shelling out two types of frames with very different origins: the "quantitative or demoscopic mode" and the "qualitative or mediatic mode" of framing. The first can be built through surveys and the second through the analysis of media content. As we will defend in the following pages, these are two complementary forms that offer, working together, a much more complete perspective of the functioning of the framings in society.

Keywords: Agenda setting, framing, mass media, polls, electoral campaign, Spain. 


\section{LA "VÍA MEDIÁTICA" Y LA “VÍA DEMOSCÓPICA" EN LA RECONS- TRUCCIÓN DE ENCUADRES}

La reconstrucción de los encuadres que circulan en una sociedad se ha realizado, principalmente, a través de la acción de los medios de comunicación. Sin embargo, en las encuestas post electorales de las elecciones generales españolas de 2008 y 2011 el CIS ha introducido una serie de preguntas para conocer el grado de aceptación de algunos encuadres de agenda pública.

¿Cuál de estas dos formas es capaz de dar cuenta de manera más fidedigna del proceso de construcción y aceptación/rechazo de encuadres? Ambas formas presentan fortalezas y limitaciones.

La "vía demoscópica" ofrece certidumbre acerca del grado de aceptación o rechazo a los encuadres enfrentados sobre un determinado tema de debate (lucha contra ETA, adopción homosexual, estado del bienestar...). Pero, por otra parte, siempre existe la duda razonable de la procedencia de estos encuadres. ¿De dónde los ha obtenido el CIS? ¿Qué métodos ha utilizado para objetivarlos? Probablemente procedan de la siempre inconcreta "cultura política" de cada país.

La "vía mediática" reconstruye encuadres de una manera mucho más dinámica y cambiante, insertada en el propio frenesí de la campaña electoral. Estos encuadres entran en la agenda, se adaptan a las circunstancias, se relacionan con los actores políticos y triunfan/son derrotados con una velocidad vertiginosa. Sin embargo, la metodología cuantitativa para objetivarlos no deja de presentar lagunas en la recogida y procesamiento de los datos.

Resulta probable también que los encuadres de procedencia mediática se encuentren más relacionados con la polarización partidista, mientras que los de corte ciudadano (encuestas) obedezcan a actitudes políticas más profundas y arraigadas.

El presente artículo intentará analizar de cerca estas dos vías de reconstrucción de encuadres poniendo de manifiesto su utilidad y limitaciones en los contextos concretos de las campañas electorales de 2008 y 2011 en España.

\section{LA MEDIATIZACIÓN DE LA REALIDAD}

Los medios de comunicación, desde la irrupción de la sociedad de masas a principios del siglo $X X$, se han convertido en el principal elemento mediante el cual los ciudadanos conocen el entorno político que les rodea.

La sociedad de masas supone un distanciamiento entre el individuo y los diferentes acontecimientos de interés social. La comunidad se amplía, transciende 
el ámbito de lo local, y el sistema de comunicación de los estados tendrá ante sí la tarea de acercar la vida comunitaria a la vida cotidiana de los individuos. Las comunidades, cada vez más, se "imaginan" (Anderson, 2006) gracias a los me$\operatorname{dios}^{2}$.

Desde el momento en que la sociedad se compone de individuos que no asisten a lo que sucede, los medios de comunicación adquieren una importante labor. Y de ahí su denominación: los medios "median" entre la realidad y lo que sucede (Iyengar y McGrady, 2007). Y una de las explicaciones más convincentes de cómo tiene lugar el proceso de mediatización de la realidad nos la proporciona la teoría del framing.

Gracias a los medios los ciudadanos conocen su entorno y se conocen a sí mismos, de modo que las noticias que los medios publican se convierten en una suerte de ventanas abiertas al mundo a través de la cual los ciudadanos aprenden y tienen constancia de sus propias instituciones, líderes políticos, estilos de vida y de otras naciones y pueblos (Tuchman, 1978).

Pero observar el mundo a través del marco de una venta supone una diferencia notable con el propio mundo. Una cosa es la realidad y otra un determinado relato sobre esa realidad. Es decir, todo proceso de enmarcado implica la inclusión de unos aspectos y la exclusión de otros (Etmann, 1993). Supone dar importancia a unas cuestiones sobre otras que, en muchas ocasiones, quedarán fuera del relato noticioso ${ }^{3}$.

La teoría del framing, que parte de la sociología de Goffmann (2006), estudia el modo en que funciona la cognición humana. Los individuos estructuran la realidad siguiendo marcos de interpretación o referencia. Estos marcos dotan de significado a los objetos sociales que rodean al sujeto. Los individuos estructuran lo que les rodea siguiendo marcos de sentido, una forma más o menos económica de utilizar una especie de "depósitos de información" para interpretar la cambiante realidad.

Los estudiosos de la comunicación pronto se dieron cuenta que la idea de marco tenía un excelente encaje en la investigación de medios (Gitlin, 1980) y que resultaba un buen complemento para la teoría de agenda setting.

En su formulación original (McCombs y Shaw, 1972) la agenda setting resultaba un tanto ingenua: Se sostenía que los medios de comunicación de masas se limitaban a seleccionar e imponer una serie de temas que, a la postre, conforma-

\footnotetext{
2 En el argumento original de Anderson (2006) la "imaginación" de comunidades tenía lugar mediante la capacidad de transmisión de conocimientos y mitos fundacionales que proporciona el libro impreso. Nuestra versión sustituye la imprenta por los medios de comunicación que, en realidad, elaboran mitos y relatos compartidos de forma muy similar. Si acaso de manera más potente.

${ }^{3}$ Esta diferencia en los encuadres que presentan los medios se relaciona con conceptos como la polarización y el partidismo. Para una visión sobre los tipos de partidismo y sus respectivos orígenes en España acúdase a González, Rodríguez y Castromil (2010).
} 
rían la agenda de prioridades ciudadanas y darían lugar al surgimiento de la opinión pública.

Sin embargo, años después, se terminaría por aceptar que los medios, además de seleccionar, encuadran los temas de debate que ofrecen a sus audiencias (McCombs y Shaw, 1993). Esto es, los medios de comunicación seleccionan temas definiendo una cuestión de debate público como problemática, interpretan sus causas y consecuencias, hacen una evaluación moral de lo sucedido y, por lo general, ofrecen a sus públicos una recomendación o pauta de actuación (Entman, 1993).

Los encuadres mediáticos, de este modo, suelen contener atribuciones de responsabilidad (Iyengar, 1991) que, en numerosas ocasiones, señalan directamente a los gestores públicos.

Esta característica de los encuadres que construyen los medios de comunicación resulta de especial interés en el seno de una campaña electoral, tal y como comprobaremos en las páginas que siguen. Entenderemos aquí por campaña el momento de máxima pugna entre encuadres antagónicos por el favor del público votante.

El interés y la aplicación de la teoría del framing en España son mucho más recientes. Un crucial paso adelante en este tipo de estudios se produce cuando en 2008 el CIS decide incluir en su encuesta postelectoral una serie de preguntas con encuadres sobre temas de debate que se consideran relevantes.

A partir de una serie de investigaciones cualitativas previas financiadas por el propio CIS (González, Rodríguez y Castromil, 2012; Castromil y Rodríguez, 2011; Bouza et al 2013) se incluyen en los estudios 2757 (post electoral 2008) y estudio 2920 (post electoral 2011) una serie de encuadres antagónicos sobre los principales temas de debate público en la campaña electoral.

Tal y como veremos en las páginas que siguen, a partir de este momento se dispone en España de material empírico suficiente como para conocer la circulación de temas y encuadres en el seno de una campaña electoral y su grado de aceptación por parte de la ciudadanía. A la "vía mediática" se le unía la "vía demoscópica".

\section{DISEÑO DE LA INVESTIGACIÓN}

Nuestra investigación tiene dos partes: En primer lugar, analizaremos la codificación y principales características de las preguntas sobre encuadres que ha incluido el CIS en las campañas de 2008 y 2011. Veremos cómo los encuadres tienen mucho que ver con la ideología y el recuerdo de voto. Se estudiará como inscribirse en la órbita de uno $u$ otro encuadre sobre un tema de debate deter- 
minado tiene mucho que ver con valores que apuntan a visiones de vida de corte conservadora o progresista.

Por otro lado, ofreceremos al lector datos que las encuestas del CIS no muestran: la procedencia de tales encuadres ("vía mediática"). En primer lugar, ¿por qué esos temas y no otros? Y, una vez en posesión de una agenda de temas de campaña, ¿por qué esos encuadres y no otros?

FIGURA 1. "Vía mediática" y "vía demoscópica" para el estudio de encuadres

\section{Vía mediática / nivel macro Vía demoscópica / nivel micro}

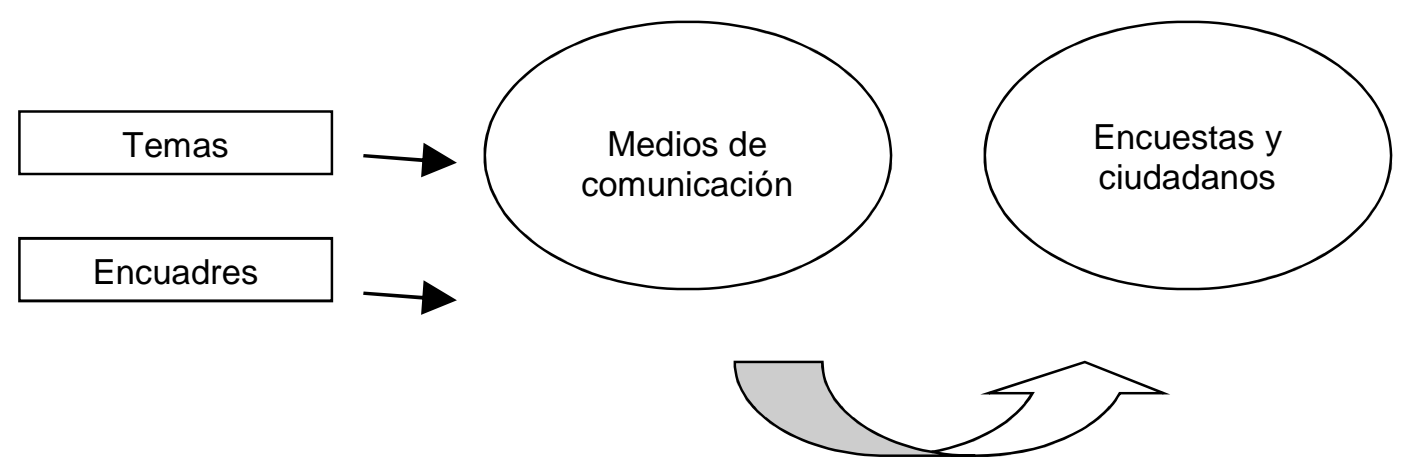

Fuente: Elaboración propia

El origen de los encuadres ofrecidos a los ciudadanos se encuentra en los datos producidos a partir de dos proyectos de investigación basados en seguimiento de prensa y grupos de investigación'. Desgranaremos las principales cuestiones metodológicas y de procedimiento que permitieron al CIS ofrecer encuadres sobre temas de debate en 2008 y 2011.

\section{LA “VÍA DEMOSCÓPICA O CUANTITATIVA” DEL ENCUADRE}

La incorporación de los encuadres sobre los principales temas de debate en las encuestas post electorales del CIS es algo, como acabamos de ver, muy reciente.

\footnotetext{
4 "Agenda de los medios y agenda ciudadana: Análisis temporal desde un enfoque cualitativo" y "Voto racional y agenda mediática. Propuesta de seguimiento de la legislatura a través de grupos experimentales".
} 
De las once elecciones generales celebradas en España hasta 2011 sólo disponemos de datos para las dos últimas (2008 y 2011).

Este reciente interés demuestra hasta qué punto la teoría del framing está ganando espacio a la hora de estudiar la cognición ciudadana en materia política. $\mathrm{Y}$, como hemos comentado más arriba, manejar este enfoque implica tener muy en cuenta los flujos comunicativos, especialmente durante el desarrollo de una campaña electoral.

La metodología que ha seguido el CIS ha variado poco en 2011 con respecto a 2008. En ambas elecciones disponemos de datos sobre el grado de adhesión a encuadres (antagónicos, enfrentados) sobre un buen número de temas de debate público y, sólo en 2008, se establece una jerarquía de importancia entre tales temas.

Es decir, sólo para 2008 podemos conocer, además de la adhesión a los encuadres, la relevancia general que el tema tiene dentro del orden de prioridades de cada ciudadano. No se trata de una cuestión menor. Se puede dar el caso, por ejemplo, de un votante que tenga muy clara (intensa) su adscripción a uno de los dos polos de un encuadre pero que, sin embargo, reconozca que el tema en cuestión resulta secundario en comparación con otros sobre los que su posición en los encuadres no es tan clara. Estas cuestiones sólo es posible conocerlas para la campaña electoral de $2008^{5}$.

Examinaremos ahora brevemente del proceso de reconstrucción de encuadres que ha llevado a cabo del CIS en 2008 y 2011. El primer paso será elegir los temas de debate que más interesa conocer en la campaña. En 2008 se pidió la opinión al votante sobre inmigración, servicios públicos, adopción homosexual, terrorismo, tolerancia religiosa y organización territorial del estado. En 2011 se repiten estas cuestiones con la única salvedad de la introducción de un nuevo tema (democracia directa) y la modificación de otro (terrorismo, sustituido por proceso de paz o fin del principal foco de terrorismo en España, la banda secesionista vasca ETA).

A continuación, el CIS construye encuadres por medio de la formulación de dos posturas extremas en cada uno de estos temas, ofreciendo al encuestado la posibilidad de adherirse a una de las dos versiones enfrentadas en distinto grado de intensidad. El 0 expresa el máximo acuerdo a un polo del encuadre, el 5 la equidistancia y el 10 la adhesión máxima a la otra forma de entender el problema.

\footnotetext{
${ }^{5}$ Así, los temas más importantes para los electores españoles serán el terrorismo $(92,4 \%)$ y los servicios públicos $(85,5 \%)$ y los más residuales la adopción homosexual $(31,3 \%)$ y la tolerancia religiosa $(22,7 \%)$. Más información en la Tabla A1 de los Anexos.
} 
TABLA 1. Temas encuadrados durante la campaña (CIS, 2008 y 2011)

\begin{tabular}{cc}
\hline 2008 & 2011 \\
\hline Inmigración & Inmigración \\
Servicios públicos & Servicios públicos \\
Adopción homosexual & Adopción homosexual \\
Lucha antiterrorista & Proceso de paz \\
Tolerancia religiosa & Democracia directa \\
\hline
\end{tabular}

Fuente: CIS (Estudios 2757 y 2920)

\subsection{Temas y encuadres en las encuestas (España 2008 y 2011).}

La figura 2 describe el camino seguido por el CIS a la hora de ofrecer encuadres antagónicos a los votantes en las elecciones generales españolas de 2008 y 2011. Explica el paso de la selección de temas (agenda setting) a su encuadre (framing).

Todos estos temas y sus respectivos encuadres antagónicos (0 vs 10) contienen en su interior una clara atribución de responsabilidad $\mathrm{y}$, generalmente, apuntan hacia el partido en el poder. En este caso, tanto en 2008 como 2011, el partido en el gobierno era el Partido Socialista Obrero Español y, por lo tanto, las atribuciones de responsabilidad tomarán la forma de premio a su gestión (reelección) o castigo (voto por el challenger, el Partido Popular). Revisemos tema a tema el contenido polarizado de los encuadres.

En el caso de la inmigración (2008 y 2011) las visiones antagónicas tienen que ver con la consideración de que la inmigración constituye un fenómeno que, ante todo, debe ser considerado positivo y enriquecedor para la convivencia. Se trata de la visión más progresista de los flujos migratorios.

Los individuos que comparten esta visión se ubicaron en valores que van desde el 0 (acuerdo máximo) al 4 (acuerdo más leve). Por otro lado, se ofrece al elector la visión que considera la inmigración como un peligro para la convivencia: 6 sería el acuerdo más leve y 10 el máximo. Se trata de la visión más restrictiva y/o xenófoba ${ }^{6}$.

\footnotetext{
${ }^{6}$ Nuestra recodificación consistió en agrupar los valores 0-2 y 8-10 como los de apoyo máximo a cada uno de los extremos, en los lugares 3-4 y 6-7 a aquellos encuestados con un apoyo más dubitativo y el valor 5 se mantuvo como la equidistancia. Esta recodificación se mantiene en el resto de temas tratados en el estudio.
} 
FIGURA 2. Selección y encuadre CIS (2008 y 2011)

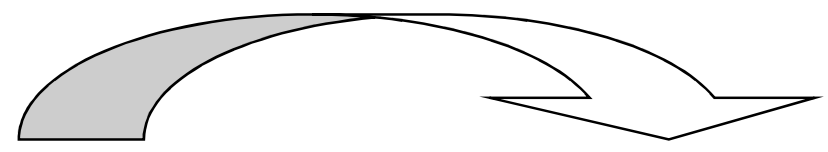

\begin{tabular}{|c|c|}
\hline $\begin{array}{c}\text { Selección de temas } \\
\text { (Aaenda settina) }\end{array}$ & $\begin{array}{c}\text { Encuadre de temas } \\
\text { (Framina) }\end{array}$ \\
\hline Inmigración (2008 y 2011) & $0=$ Positivo convivencia $10=$ Peligro \\
\hline Servicios públicos (2008 y 2011) & $\begin{array}{l}0=\text { Mejorar servicios } 10=\text { Menos im- } \\
\text { puestos }\end{array}$ \\
\hline Adopción homosexual (2008 y 2011) & $0=$ Permitir $10=$ No permitir \\
\hline $\begin{array}{l}\text { Terrorismo (2008) / Proceso de pacifi- } \\
\text { cación (2011) }\end{array}$ & $\begin{array}{l}0=\text { Vía policial } / \text { No dialogar } 10 \text { = Diá- } \\
\text { logo / diálogo }\end{array}$ \\
\hline Tolerancia religiosa (2008) & $0=$ Valores propios $10=$ Tolerancia \\
\hline Democracia directa (2011) & $0=$ Ciudadanos $10=$ Elites políticas \\
\hline
\end{tabular}

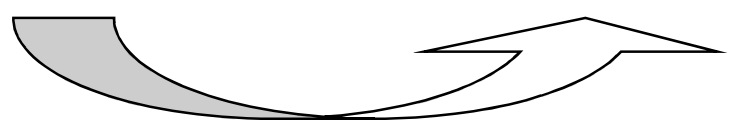

Fuente: Elaboración propia

El tema de debate servicios públicos (2008 y 2011) se centra en la división social que puede observarse entre aquellos que apuestan por un estado del bienestar fuerte y completo, aún a costa de unos impuestos elevados (postura más socialdemócrata o progresista). La otra postura sería la de aquellos que valoran, sobre todas las circunstancias, la bajada de la presión fiscal, aún a costa de peores servicios públicos (apuesta liberal-conservadora).

Los encuadres enfrentados sobre adopción homosexual (2008 y 2011) y tolerancia religiosa (2008) tienen que ver con cuestiones morales muy polarizantes o posicionales (Maravall, 2008). Los más permisivos hacia la posibilidad de que las personas homosexuales puedan adoptar niños y formar una familia (progre- 
sistas) tenderán a ubicarse en las proximidades del valor 0 , mientras que los contrarios a tal posibilidad lo harán en el 10 (conservadores).

La escala de intensidad en los encuadres sobre tolerancia religiosa cambia y ahora la visión más progresista (más tolerancia) se sitúa en el 10 y la más conservadora (reivindicar y hacer prevalecer los valores propios de la comunidad frente a otros externos) en el 0.

En materia de terrorismo en sus dos variantes (2008: lucha contra ETA; 2011: gestión del proceso de paz) los encuadres se dividen entre los partidarios del diálogo (valores cercanos al 10) y los que creen que la vía policial y la ausencia de diálogo deben ser las políticas preferentes del gobierno en materia antiterrorista (valores cercanos al 0).

En las elecciones generales de 2011, debido a las movilizaciones ciudadanas recientes y al aumento de la desafección política7, el CIS introduce un encuadre, ausente en 2008, que se fija en la conveniencia (visión progresista) o no (visión conservadora) de aumentar el los grados de democracia directa (2011) en el sistema político español.

Los partidarios de incrementar la posibilidad de que sean los propios ciudadanos los que tomen las decisiones políticas se mueven en torno al valor 0 y aquellos otros que apuestan por mantener la preeminencia de la elites políticas se sitúan, en diferente grado de intensidad, en valores próximos al $10^{8}$.

\subsection{Recepción de encuadres de campaña (España 2008 y 2011)}

Una vez conocidas las posturas antagónicas sobre cada uno de los temas seleccionados por el CIS en las campañas electorales de 2008 y 2011 pasamos a ver ahora la ubicación de los ciudadanos en cada uno de ellas. Es decir, se comprobará aquí si estas ubicaciones han funcionado o no o, dicho de otra manera, si los votantes españoles conocen estas divisiones y son capaces de adscribirse a alguno de los dos polos en conflicto.

En temas migratorios los españoles mayoritariamente se acogen al encuadre progresista (multiculturalismo) tanto en 2008 como en 2011, cuando la crisis económica ya estaba plenamente instalada. Los partidarios del encuadre progresista (48,3\% en 2008 y 46,7\% en 2011) casi duplican a los que optan por considerar la inmigración como peligrosa para la convivencia $(27,8 \%$ en 2008 y $28,8 \%)$.

\footnotetext{
7 Según datos de la propia encuesta post electoral que nos sirve de base en 2011 la desafección ciudadana hacia el Parlamento y los partidos políticos alcanza al 51,2\% del total de la muestra (Tabla A2 en los anexos).

8 El CIS incluye en las post electorales de 2008 y 2011 un tema muy recurrente en la política española como es la organización territorial del estado. La polémica se centra entre los partidarios de una mayor centralización del estado, los equidistantes y aquellos otros más en la línea de aumentar la descentralización. Por la diferente formulación de los encuadres esta cuestión quedará fuera de nuestro objeto de estudio.
} 
La procedencia cuantitativa y cualitativa de los encuadres sociales: el caso de las elecciones generales...

TABLA 2. Recepción ciudadana a encuadres de campaña (\%)

\begin{tabular}{|c|c|c|c|c|c|c|c|c|c|c|}
\hline & \multicolumn{2}{|c|}{ Inmigración } & \multicolumn{2}{|c|}{$\begin{array}{l}\text { Servicios Pú- } \\
\text { blicos }\end{array}$} & \multicolumn{2}{|c|}{$\begin{array}{c}\text { Adopción } \\
\text { homosexual }\end{array}$} & \multicolumn{2}{|c|}{$\begin{array}{l}\text { Terrorismo / } \\
\text { Proceso paz }\end{array}$} & \multicolumn{2}{|c|}{$\begin{array}{c}\text { Religión / } \\
\text { Democracia }\end{array}$} \\
\hline & $\begin{array}{c}2008 \\
\%\end{array}$ & $\begin{array}{c}2011 \\
\%\end{array}$ & $\begin{array}{c}2008 \\
\%\end{array}$ & $\begin{array}{c}2011 \\
\%\end{array}$ & $\begin{array}{c}2008 \\
\%\end{array}$ & $\begin{array}{c}2011 \\
\%\end{array}$ & $\begin{array}{c}2008 \\
\%\end{array}$ & $\begin{array}{c}2011 \\
\%\end{array}$ & $\begin{array}{c}2008 \\
\%\end{array}$ & $\begin{array}{c}2011 \\
\%\end{array}$ \\
\hline $\begin{array}{c}\text { Muy de acuer- } \\
\text { do } \\
(0-2)\end{array}$ & 28,9 & 29,1 & 29,0 & 24,6 & 36,7 & 42,8 & 35,2 & 45,8 & 10,1 & 17,6 \\
\hline $\begin{array}{c}\text { Bastante de } \\
\text { acuerdo } \\
(3-4)\end{array}$ & 19,4 & 17,6 & 23,4 & 20,9 & 10,1 & 9,6 & 9,3 & 9,0 & 8,8 & 14,4 \\
\hline $\begin{array}{c}\text { Equidistante } \\
(5)\end{array}$ & 20,6 & 21,2 & 24,8 & 27,1 & 11,9 & 12,6 & 15,1 & 9,9 & 13,8 & 33,8 \\
\hline $\begin{array}{c}\text { Bastante de } \\
\text { acuerdo } \\
(6-7)\end{array}$ & 13,8 & 15,2 & 9,5 & 11,7 & 7,8 & 7,5 & 11,0 & 9,1 & 13,8 & 14,4 \\
\hline $\begin{array}{c}\text { Muy de acuer- } \\
\text { do } \\
(8-10)\end{array}$ & 14,0 & 13,7 & 6,8 & 9,2 & 26,8 & 20,8 & 22,5 & 19,0 & 49,7 & 14,1 \\
\hline NS/NC & 3,4 & 3,2 & 6,5 & 6,5 & 6,8 & 6,7 & 6,8 & 7,2 & 3,7 & 5,6 \\
\hline
\end{tabular}

Fuente: CIS (Estudios 2757 y 2920) N 2008: 6083 N 2011: 6082

La inmigración ha sido una cuestión escasamente tratada en las campañas de 2008 y 2011 y siempre desde una postura (en los medios y en la clase política) muy "políticamente correcta". Es decir, no triunfan en España, al menos de momento, discursos políticos de corte populista y xenófobo que conectan inmigración y descontento económico con el único fin de ganar votos. Esto no sucede simplemente porque los encuadres conservadores no tienen demasiados adeptos.

Los encuadres sobre el mantenimiento de un potente estado de bienestar a cambio de una mayor presión fiscal (socialdemócratas, estatistas, progresistas) frente a la alternativa liberal-conservadora que opta por menores impuestos a costa de peores y menos universales servicios públicos se resuelve con una holgada victoria del encuadre progresista. 52,4\% (2008) y 45,5\% (2011) frente al $16,3 \%$ (2008) y 20,9\% (2011) de los conservadores.

Sobre la conveniencia o no de otorgar a las parejas homosexuales el derecho a adoptar niños y formar una familia la cosa está algo más reñida, aunque siguen prevaleciendo los encuadres permisivos-progresistas $(46,8 \%$ en 2008 y $52,4 \%$ en 2011) sobre los restrictivos-conservadores (34,6\% en 2008 y $28,3 \%$ en 2011). En el transcurso de los tres años que median entre 2008 y 2011 parecen reforzarse las posiciones liberales sobre las conservadoras. 
Otros dos temas político-morales ofrecen, sin embargo, resultados algo distintos. En terrorismo se imponen los encuadres contrarios al diálogo, la negociación y favorables a la lucha policial (44,5\% en 2008 y 54,8\% en 2011, postura conservadora) frente a las posturas más pro diálogo (33,5\% en 2008 y $28,1 \%$ en 2011, postura progresista). Los partidarios de la negociación parecen ir perdiendo fuelle con el alto al fuego definitivo de la banda terrorista ETA.

El encuadre progresista se impone, sin embargo, de manera muy clara cuando de lo que se trata es de optar por la defensa de la libertad individual (63,5\% 2008) frente a la opción de defender de manera prioritaria los valores propios (18,9\% 2008). Mucho más equilibrada se encuentra la relación de fuerzas en la cuestión de la implicación ciudadana en la toma de decisiones políticas $(32,0 \%$ 2011) frente a la opción de dejar en las manos de políticos profesionales las labores de gobierno $(28,5 \%)$.

Como resumen a este comentario sobre la recepción de encuadres en las campañas españolas de 2008 y 2011 hay que destacar el bajísimo número de ciudadanos que o no saben o deciden no hacer pública su adscripción. Sólo entre un $3 \%$ y un $7 \%$ no manejaron, de una forma u otra, estos encuadres. Parece, por lo tanto, que funcionan.

\section{LA “VÍA MEDIÁTICA O CUALITATIVA” DEL ENCUADRE}

Si lo que hemos convenido en denominar "vía demoscópica" del encuadre tiene la ventaja, como hemos visto, de poder conocer en detalle la composición sociopolítica de los ciudadanos que se van distribuyendo en su escala de intensidad polarizada la "vía mediática" supone algo así como "el origen de todo".

¿Por qué el CIS ha seleccionado la inmigración, el terrorismo y la adopción homosexual, por ejemplo, y no otros temas del, en principio, extensísimo repertorio de cuestiones de debate? ¿Por qué la participación ciudadana en política es un tema exclusivo de la campaña electoral de 2011 y por qué la tolerancia religiosa lo es de la de 2008?

En corto, ¿cuáles han sido los criterios que se han seguido para seleccionar unos temas y no otros y unos encuadres y no otros? La "vía mediática" del encuadre permite responder a todas estas interesantes preguntas.

La estrategia que se suele emplear, y que ha sido la que ha dado origen a los encuadres propuestos por el CIS a sus encuestados ${ }^{9}$, es la de realizar un seguimiento de medios durante un período determinado. El objetivo será objetivar la denominada agenda mediática que, según la teoría de agenda setting, los ciuda-

\footnotetext{
${ }^{9}$ Información más detallada en González, Rodríguez y Castromil (2012).
} 
danos van a conocer y debatir, generándose así la opinión pública (McCombs y Shaw, 1972).

En nuestro caso realizamos un seguimiento de medios de comunicación desde la primavera de 2006 hasta las elecciones generales de 2008 (primer estudio) y durante la mayor parte de la legislatura 2008-2011. El seguimiento se centró en la denominada "prensa de referencia", es decir, en las portadas de los principales diarios españoles de tirada nacional (El País, El Mundo y Abc, principalmente).

En la primera parte de nuestro estudio (2006-2008) se realizaron un total de cinco reuniones de grupos de discusión. Será en este estudio cuando se localizarán la mayor parte de temas de debate que luego se repetirán en 2011 con la única excepción, como hemos visto, del tema participación ciudadana en política.

En las reuniones (primavera 2006, otoño 2006, pre electoral locales 2007, pre electoral 2008 y post electoral 2008) se ofrecieron a los grupos ${ }^{10} \operatorname{los}$ principales temas de debate que la prensa de referencia estaba tratando en ese momento. Se les permitió un debate cómodo y flexible sobre esos temas.

Los focus group nos permitieron comprobar que los encuadres que el equipo de investigación al cargo del seguimiento de medios había reconstruido realmente circulaban en la sociedad. Nuestros grupos manejaban tales encuadres antagónicos con un grado de fidelidad pasmoso a los formulados por la prensa de referencia.

A pesar de no estar nuestros grupos integrados por lectores objetivos de prensa de referencia (clases medias urbanas), estos ciudadanos conocían los temas de la prensa de referencia, tenían una opinión formada sobre ellos y tal opinión pivotaba sobre la base de los encuadres que esa misma prensa de referencia había construido y que nosotros, como equipo investigador, habían reconstruido.

Parecía evidente que estos temas de agenda y sus respectivos encuadres se habían "traspasado" desde la prensa de referencia que los había originado hacia otros lugares como la prensa gratuita (que se regala en los principales puntos de transporte público de las ciudades), las radios y, sobre todo, las televisiones.

De este modo, la combinación de datos procedentes del seguimiento de medios y de los grupos de discusión nos permitió comprobar la corrección y potencia de la reconstrucción de la agenda mediática y, sobre todo, de los encuadres antagónicos que la prensa de referencia estaba construyendo.

\footnotetext{
${ }^{10}$ Por cuestiones de espacio no entraremos en cuestiones metodológicas referentes a la composición de grupos y a las dinámicas de las reuniones que, por otra parte, se encuentran detalladas en González, Rodríguez y Castromil (2012).
} 
El carácter antagónico de los encuadres que detectamos y nuestros grupos confirmaron se sitúa en la línea del modelo de pluralismo polarizado que Hallin y Mancini (2004) han descrito para España entre otros países del sur de Europa. Estamos ante un sistema de relación entre medios y política marcado por el partidismo, la escasa independencia del periodismo, la intrusión del estado en la ecología de medios y la escasa lectura de prensa, entre otros factores.

\subsection{Temas y encuadres en los medios (España 2008 y 2011)}

El período 2006-2008 estuvo dominado por el tema de debate terrorismo y por un encuadre muy diferente según el periódico de referencia que se tratase. La prensa progresista (diario El País) encuadró el acontecimiento muy en la línea del Gobierno socialista de José Luis Rodríguez Zapatero: se trataba de un proceso de paz con el objetivo de la erradicación total del terrorismo.

La prensa conservadora de oposición al Gobierno (El Mundo y Abc, entre otros) encuadra el tema terrorismo en términos de debilidad y cesión al chantaje de los terroristas.

Poco a poco este tema de debate fue perdiendo importancia, sobre todo a partir de la segunda legislatura del PSOE en el poder (2008-2011) debido, entre otras cosas, al cese definitivo de la actividad armada de la banda terrorista ETA (octubre 2011). A partir de ese momento, tal y como recogen los encuadres que ofrece el CIS a sus encuestados de 2011, la cuestión terrorista varía: de la mejor forma de luchar contra ETA (diálogo y policía vs sólo policía) se pasa al mejor modo de gestionar el horizonte abierto tras la desaparición de la violencia (negociación vs no negociación).

La inmigración es un tema mucho más presente en las preocupaciones de nuestros grupos de discusión que en los medios que hemos seguido. La información publicada sobre inmigración se refiere a aspectos mucho menos polémicos como la información sobre entrada ilegal de inmigrantes en el país. Se observa, por lo tanto, un cierto aire de información "políticamente correcta".

A pesar de ellos fuimos capaces de objetivar las dos visiones que sobre el fenómeno migratorio exponíamos en el anterior epígrafe: la entrada de inmigrantes como algo que beneficia a la sociedad, caminando así hacia una comunidad multicultural (encuadre progresista, PSOE y El País) o una visión restrictiva de problema de convivencia.

Con la irrupción de la crisis económica (a partir de las elecciones de 2008) se observa en los medios una creciente crisis del encuadre progresista de la inmigración, muy en la línea de ruptura de encuadre o misframing descrito por Goffman (2006: 359). Y es que, tal y como lo integrantes de nuestros grupos de dis- 
cusión estaban indicando, la visión de los inmigrantes caminaba hacia un encuadre de competencia con los nacionales por los puestos de trabajo.

El tema de debate adopción homosexual arranca de la polémica suscitada en España a raíz de la legalización del matrimonio homosexual (julio 2005) y el rechazo que suscitó en círculos políticos (Partido Popular en la oposición), sociales (Iglesia Católica y otros grupos fundamentalistas) y mediáticos (diario El Mundo y, sobre todo, Abc) conservadores. La cuestión de la adopción por parte de los gays supone, por lo tanto, una continuación del tema.

Otro tema también de corte moral será la cuestión de la tolerancia religiosa. Se trata de un tema menor y circunscrito sólo a 2008 (de ahí que el CIS lo elimine en su post electoral de 2011) que viene a incidir en la división progresista/conservador a la que se refería la adopción homosexual.

Exclusivo de unas únicas elecciones, aunque ahora de las de 2011, es el tema de debate que hace referencia al grado de participación ciudadana que debería desempeñar el ciudadano corriente en una democracia sana. Sin duda, la inclusión de esta cuestión tiene que ver con el clima político y mediático de falta de representación del ciudadano corriente que venía denunciando desde mayo de 2011 el movimiento del 15M⒒

\section{DISCUSIÓN: DOS VÍAS COMPLEMENTARIAS}

El grado de aceptación de encuadres antagónicos sobre los principales temas de debate de una campaña electoral (o estructurales en una sociedad) se conocen a través de su inclusión en las encuestas. Estos encuadres se vienen incluyendo en las encuestas post electorales del CIS desde 2008, lo que demuestra una conciencia creciente de la importancia de los medios de comunicación a la hora de organizar las actitudes políticas de los ciudadanos.

Esto es así porque los diferentes encuadres mediáticos apuntan a tradiciones políticas diferentes y sus respectivas opciones partidistas. Para la realidad española nos hemos referido al mundo progresista (o universo significativo de la izquierda) y conservador (o significante de derecha) y del Partido Socialista Obrero Español (progresismo, centro-izquierda) o Partido Popular (conservadurismo, centro-derecha).

Junto a esta vía de encuadre se sitúa otra mucho más extendida en los estudios de comunicación, la "vía mediática o vía cualitativa". Por este medio es posible obtener una riqueza quizá mayor en los matices del encuadre y descubrir los entresijos de la polarización político-mediática que opera en sistemas como el español.

${ }^{11}$ Más detalles en Castromil y Resina (2013). 
Una combinación de ambas vías de encuadre ofrece una visión de conjunto que incluye sesgos mediáticos y actitudes ciudadanas. Ojalá el CIS continúe con su apuesta por la inclusión en sus post electorales de los encuadres. Si es así, el legado de Fermín Bouza habrá valido la pena. Como diría él, siempre "palante".

\section{BIBLIOGRAFÍA}

Anderson, B (2006 V. O 1983): Comunidades imaginadas. Reflexiones sobre el origen y la difusión del nacionalismo. México. FCE.

Bouza, F. et al (2013): "Génesis y desarrollo de los encuadres periodísticos en un sistema mediático polarizado: El caso español (2004-2008)" in A. Capdevila et al (ed): Estudios de Comunicación Política. Libro del año 2012. Madrid. Tecnos.

Castromil, A. R. y Resina, J. (2013): “La prensa tradicional en la España del 15M" en M. L. Morán (ed): Actores y demandas en España. Análisis de un inicio de siglo convulso. Madrid. Catarata.

Castromil, A. R. y Chavero, P. (2012): “Polarización política y negativismo mediático. Similitudes y diferencias en la prensa de derecha y la de izquierda en las elecciones autonómicas y municipales de 2011" Redmarka. № 8 Vol. 1.

Castromil, A. R. y Rodríguez, R. (2011): "Terrorismo con y sin tregua. Políticos, ciudadanos y medios de comunicación". TELOS. № 87.

Entman, R. (1993): “Framing: Toward Clarification of a Fractured Paradigm". Journal of Communication. Vol. 43 Issue 4.

Gitlin, T. (1980): The whole world is watching. Berkeley: University of California Press.

Goffman, I (2006. V. O. 1975): Frame Analysis: Los marcos de la experiencia. Madrid. CIS.

González, J. J. Rodríguez, R. y Castromil, A. (2012): "Dilemas metodológicos en un estudio longitudinal de la influencia de los medios sobre el voto" in D. Guinea (coord.). Trucos del oficio de investigador. Casos prácticos de investigación social. Barcelona. Gedisa. 
González, J. J. Rodríguez, R. y Castromil, A. (2012): “A Case of Polarized Pluralism in a Mediterranean Country. The Media and Politics in Spain". Global Media Journal. Mediterranean Edition 5 (1/2)

Iyengar, S. y Mcgrady, J. A. (2007): Media Politics. A Citizen's Guide. New York. W.W Norton \& Company.

Iyengar, S. (1991): Is anyone responsible? How television frames political issues. Chicago. University of Chicago Press.

Maravall, J. M (2008): La confrontación política. Madrid. Taurus.

Mccombs, M. y Shaw, D. (1972): “The Agenda-Setting Function of Mass Media”. The Public Opinion Quarterly Vol. 36 № 2.

Mccombs, M. y Shaw, D. (1993): “The Evolution of Agenda-Setting Research: Twenty-Five Years in the Marketplace of Ideas". Journal of Communication Vol. 43 № 2.

Tuchman, G. (1978): Making News: A Study of the Construction of Reality. New York. The Free Press.

\section{ANEXOS}

Tabla A1. Importancia de temas de debate (solo 2008)

\begin{tabular}{|c|c|c|c|c|c|}
\hline & Inmigración & $\begin{array}{l}\text { Servicios Públi- } \\
\text { cos }\end{array}$ & $\begin{array}{l}\text { Adopción homose- } \\
\text { xual }\end{array}$ & Terrorismo & Religión \\
\hline Poco o nada importante & $17,9 \%$ & $10,1 \%$ & $31,3 \%$ & $4,6 \%$ & $22,7 \%$ \\
\hline $\begin{array}{l}\text { Bastante o muy impor- } \\
\text { tante }\end{array}$ & $77,9 \%$ & $85,5 \%$ & $63,2 \%$ & $92,4 \%$ & $72,7 \%$ \\
\hline NS & $2,7 \%$ & $3,1 \%$ & $3,8 \%$ & $1,8 \%$ & $2,9 \%$ \\
\hline $\mathrm{NC}$ & $1,5 \%$ & $1,4 \%$ & $1,7 \%$ & $1,3 \%$ & $1,7 \%$ \\
\hline TOTAL & $100 \%$ & $100 \%$ & $100 \%$ & $100 \%$ & $100 \%$ \\
\hline
\end{tabular}

Fuente: CIS (Estudio 2757) N: 6083

Tabla A2. Desafección ciudadana hacia el parlamento y los partidos (solo 2011)

\begin{tabular}{lc}
\hline & $\%$ \\
\hline Desafectos & $51,2 \%$ \\
Equidistantes & $19,8 \%$ \\
Afectos & $26,0 \%$ \\
NS/NC & $3,0 \%$ \\
TOTAL & $100 \%$ \\
\hline
\end{tabular}

Fuente: CIS (Estudio 2920) N: 6082 
Tabla A3. Inmigración, ideología y recuerdo de voto (2008 y 2011)

\begin{tabular}{|c|c|c|c|c|c|c|c|c|c|}
\hline & \multicolumn{8}{|c|}{2008} \\
\hline & & Izquierda & \multicolumn{2}{|c|}{ Centro izquierda } & Centro & \multicolumn{2}{|c|}{ Centro derecha } & Derecha & TOTAL \\
\hline \multirow[t]{2}{*}{ Positiva ++} & $\%$ & $50,9 \%$ & \multicolumn{2}{|c|}{$39,1 \%$} & $21,0 \%$ & \multicolumn{2}{|c|}{$15,1 \%$} & $15,2 \%$ & $30,5 \%$ \\
\hline & RTC & $10,6^{*}$ & \multicolumn{2}{|c|}{$11,1^{*}$} & $-10,5^{*}$ & \multicolumn{2}{|c|}{$-9,3^{*}$} & $-3,6^{*}$ & - \\
\hline \multirow[t]{2}{*}{ Positiva + } & $\%$ & $15,3 \%$ & \multicolumn{2}{|c|}{$22,9 \%$} & $21,0 \%$ & \multicolumn{2}{|c|}{$16,6 \%$} & $17,9 \%$ & $20,6 \%$ \\
\hline & RTC & $-3,1^{*}$ & \multicolumn{2}{|c|}{$3,4^{*}$} & ,6 & \multicolumn{2}{|c|}{$-2,7^{*}$} & $-0,7$ & - \\
\hline \multirow[t]{2}{*}{ Equidistante } & $\%$ & $15,1 \%$ & \multicolumn{2}{|c|}{$16,5 \%$} & $27,9 \%$ & \multicolumn{2}{|c|}{$22,2 \%$} & $16,1 \%$ & $21,0 \%$ \\
\hline & RTC & $-3,4^{*}$ & \multicolumn{2}{|c|}{$-6,5^{*}$} & $8,7 *$ & \multicolumn{2}{|c|}{,8 } & $-1,3$ & - \\
\hline \multirow[t]{2}{*}{ Peligrosa + } & $\%$ & $7,8 \%$ & \multicolumn{2}{|c|}{$12,0 \%$} & $16,2 \%$ & \multicolumn{2}{|c|}{$19,8 \%$} & $13,4 \%$ & $14,1 \%$ \\
\hline & RTC & $-4,3^{*}$ & \multicolumn{2}{|c|}{$-3,4^{*}$} & $3,1 *$ & \multicolumn{2}{|c|}{$4,6^{*}$} & $-0,2$ & - \\
\hline Peligrosa ++ & $\%$ & $10,9 \%$ & & $4 \%$ & $13,8 \%$ & & & $37,5 \%$ & $13,9 \%$ \\
\hline & RTC & $-2,1^{*}$ & & $7,6^{*}$ & $-0,1$ & & & $7,3^{*}$ & - \\
\hline TOTAL & & $100 \%$ & & $00 \%$ & $100 \%$ & & & $100 \%$ & $100 \%$ \\
\hline RTC $> \pm 1,96(* *) \searrow$ & ${ }^{2}=513,0$ & 15948206 & $=16 \mathrm{Sig}$ & 000 & & & & & \\
\hline & & & & & & & & & \\
\hline & & Izquierda & Centr & izquierda & Centro & Centro & recha & Derecha & TOTAL \\
\hline Positiva ++ & $\%$ & $5,1 \%$ & & $3,7 \%$ & $8,6 \%$ & & & $8 \%$ & $30,9 \%$ \\
\hline & RTC & 9,9 & & 11,0 & $-7,1$ & & & $-4,8$ & - \\
\hline Positiva + & $\%$ & $1,1 \%$ & & $6 \%$ & $7,2 \%$ & & &, $6 \%$ & $18,5 \%$ \\
\hline & RTC & $-4,7$ & & 1,7 & 2,8 & & & $-2,1$ & - \\
\hline Equidistante & $\%$ & $2,0 \%$ & & $6 \%$ & $9,1 \%$ & & &, $9 \%$ & $21,6 \%$ \\
\hline & RTC & $-1,3$ & & $-5,9$ & 5,8 & & &,- 7 & - \\
\hline Peligrosa + & $\%$ &, $9 \%$ & & $3 \%$ & $5,8 \%$ & & & $1,0 \%$ & $15,7 \%$ \\
\hline & RTC & $-4,7$ & & 4,0 & 1,2 & & & 2,6 & - \\
\hline Peligrosa ++ & $\%$ & $1,1 \%$ & & $2 \%$ & $4,2 \%$ & & & $1,3 \%$ & $13,2 \%$ \\
\hline & RTC & $-1,5$ & & 5,4 & $-1,7$ & & & 7,0 & - \\
\hline TOTAL & & $100 \%$ & & $00 \%$ & $100 \%$ & & & $100 \%$ & $100 \%$ \\
\hline RTC $> \pm 1,96(*) X$ & $=421,64$ & $9998858 \mathrm{G}$ & 16 Sig. & & & & & & \\
\hline & & & & & & & & & \\
\hline & & 2008 & 2011 & 2008 & 2011 & 2008 & 2011 & 2008 & 2011 \\
\hline Positiva ++ & $\%$ & $37,0 \%$ & $7,8 \%$ & $16,2 \%$ & $6,5 \%$ & $23,8 \%$ & $15,8 \%$ & $29,2 \%$ & $30,1 \%$ \\
\hline & RTC & $13,6^{*}$ & $6,2^{*}$ & $-13,5^{*}$ & $-12,2^{*}$ & $-0,5$ & $6,4^{*}$ & - & - \\
\hline Positiva + & $\%$ & $20,9 \%$ & $3,9 \%$ & $18,7 \%$ & $6,0 \%$ & $19,0 \%$ & $8,2 \%$ & $20,1 \%$ & $18,2 \%$ \\
\hline & RTC & 1,6 & 0,6 & $-1,6$ & 0,1 & $-0,1$ & $-0,6$ & - & - \\
\hline Equidistante & $\%$ & $18,8 \%$ & $4,1 \%$ & $24,3 \%$ & $7,8 \%$ & $14,3 \%$ & $10,0 \%$ & $20,8 \%$ & $21,9 \%$ \\
\hline & RTC & $-3,9^{*}$ & $-1,9^{*}$ & $4,1^{*}$ & $2,2^{*}$ & $-0,7$ & $-0,4$ & - & - \\
\hline Peligrosa + & $\%$ & $12,0 \%$ & $2,7 \%$ & $19,9 \%$ & $6,5 \%$ & $28,6 \%$ & $6,6 \%$ & $15,0 \%$ & $15,7 \%$ \\
\hline & RTC & $-6,7^{*}$ & $-3,1^{*}$ & $6,5^{*}$ & $5,8^{*}$ & 1,7 & $-2,9^{*}$ & - & - \\
\hline Peligrosa ++ & $\%$ & $11,3 \%$ & $2,3 \%$ & $20,8 \%$ & $6,2 \%$ & $14,3 \%$ & $5,6 \%$ & $14,9 \%$ & $14,1 \%$ \\
\hline & RTC & $-7,9^{*}$ & $-3,3^{*}$ & $7,9^{*}$ & $7,3^{*}$ & $-0,1$ & $-4,2^{*}$ & - & - \\
\hline TOTAL & & $100 \%$ & $100 \%$ & $100 \%$ & $100 \%$ & $100 \%$ & $100 \%$ & $100 \%$ & $100 \%$ \\
\hline
\end{tabular}

Fuente: CIS (Estudios 2757 y 2920) 
La procedencia cuantitativa y cualitativa de los encuadres sociales: el caso de las elecciones generales...

Tabla A4. Servicios públicos, ideología y recuerdo de voto (2008 y 2011)

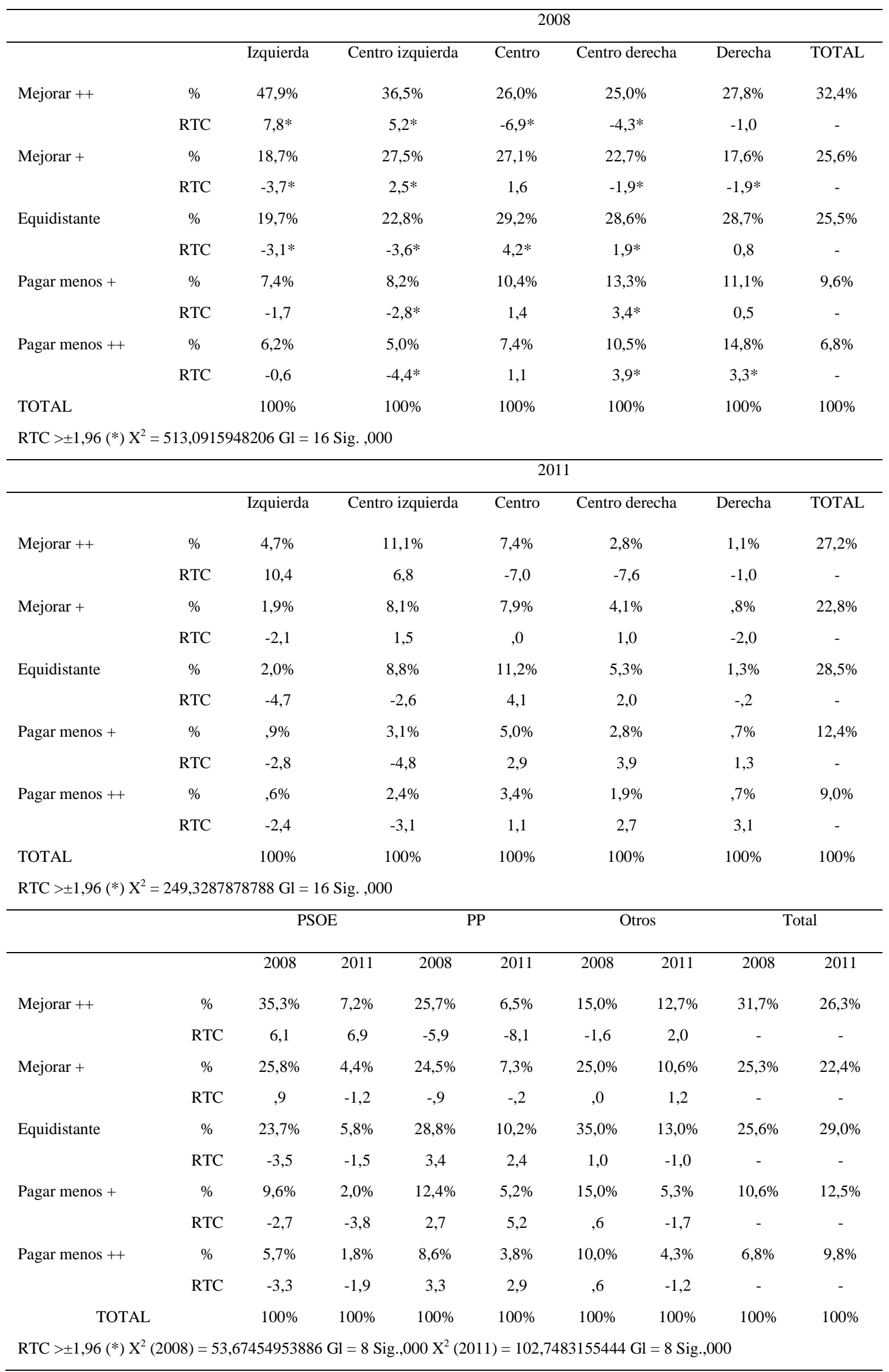

Fuente: CIS (Estudios 2757 y 2920) 
Tabla A5. Adopción homosexual, ideología y recuerdo de voto (2008 y 2011)

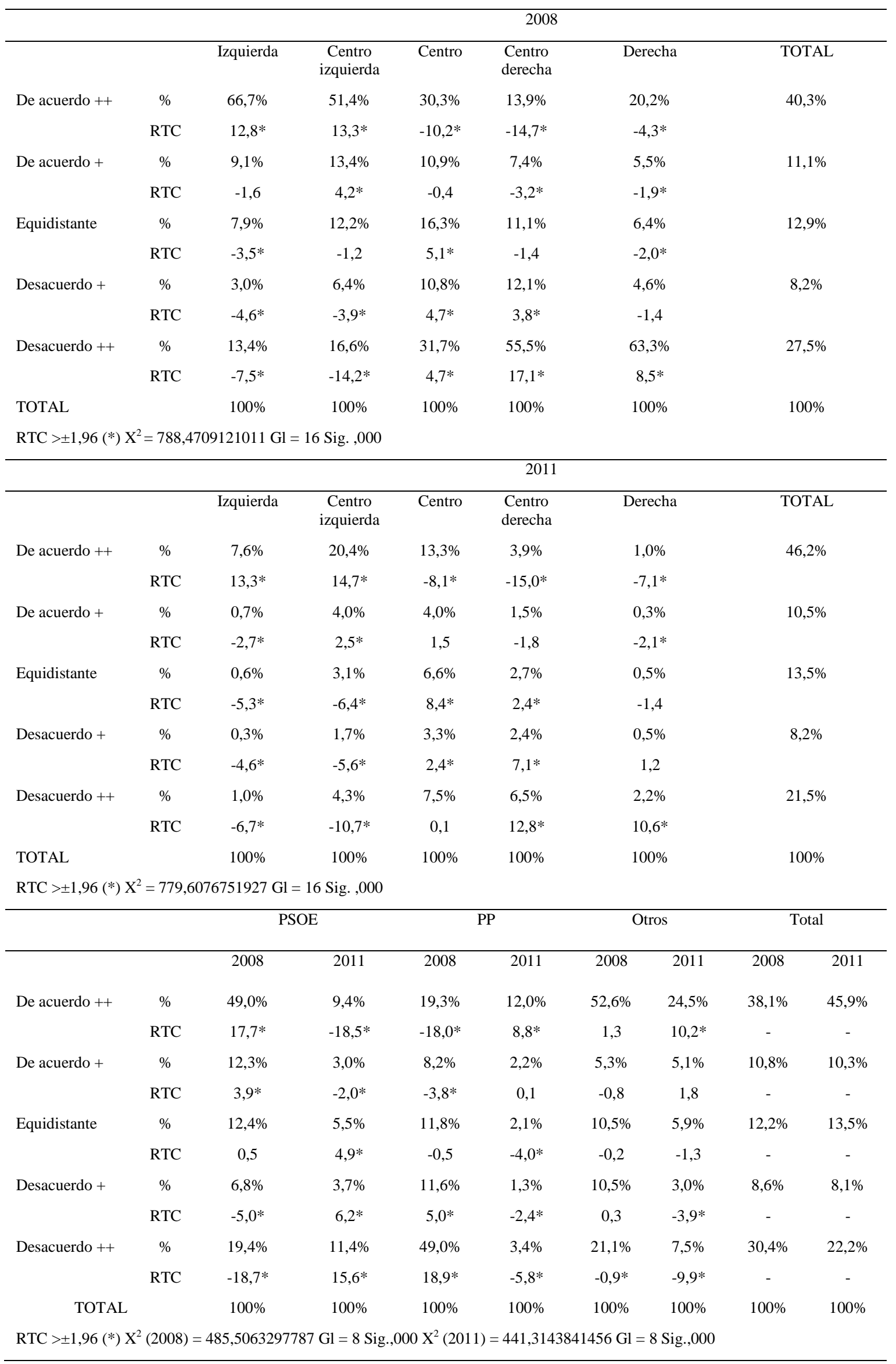


La procedencia cuantitativa y cualitativa de los encuadres sociales: el caso de las elecciones generales...

Tabla A6. Terrorismo, ideología y recuerdo de voto (2008 y 2011)

\begin{tabular}{|c|c|c|c|c|c|c|c|}
\hline & & \multicolumn{6}{|c|}{2008} \\
\hline & & Izquierda & $\begin{array}{l}\text { Centro } \\
\text { izquierda }\end{array}$ & Centro & $\begin{array}{l}\text { Centro } \\
\text { derecha }\end{array}$ & Derecha & TOTAL \\
\hline \multirow[t]{2}{*}{ Policía ++ } & $\%$ & $16,5 \%$ & $25,6 \%$ & $43,6 \%$ & $62,5 \%$ & $67,6 \%$ & $36,5 \%$ \\
\hline & RTC & $-9,8^{*}$ & $-13,2 *$ & $7,5^{*}$ & $14,7 *$ & $6,8^{*}$ & - \\
\hline \multirow[t]{2}{*}{ Policía + } & $\%$ & $3,4 \%$ & $9,5 \%$ & $11,5 \%$ & $14,8 \%$ & $9,3 \%$ & $10,2 \%$ \\
\hline & RTC & $-5,3^{*}$ & $-1,5$ & $2,1^{*}$ & $4,1^{*}$ & $-0,3$ & - \\
\hline \multirow[t]{2}{*}{ Equidistante } & $\%$ & $14,1 \%$ & $18,3 \%$ & $17,1 \%$ & $8,4 \%$ & $8,3 \%$ & $16,0 \%$ \\
\hline & RTC & $-1,2$ & $3,7^{*}$ & 1,6 & $-5,6^{*}$ & $-2,2^{*}$ & - \\
\hline \multirow[t]{2}{*}{ Diálogo + } & $\%$ & $10,7 \%$ & $15,5 \%$ & $10,6 \%$ & $6,3 \%$ & $4,6 \%$ & $12,0 \%$ \\
\hline & RTC & $-0,9$ & $6,4^{*}$ & $-2,0^{*}$ & $-4,8^{*}$ & $-2,4^{*}$ & - \\
\hline \multirow[t]{2}{*}{ Diálogo ++ } & $\%$ & $55,3 \%$ & $31,1 \%$ & $17,2 \%$ & $8,0 \%$ & $10,2 \%$ & $25,4 \%$ \\
\hline & RTC & 16,2 & 7,7 & $-9,5$ & $-10,9$ & $-3,7$ & - \\
\hline TOTAL & & $100 \%$ & $100 \%$ & $100 \%$ & $100 \%$ & $100 \%$ & $100 \%$ \\
\hline
\end{tabular}

\begin{tabular}{|c|c|c|c|c|c|c|c|c|c|}
\hline & & & & & & 011 & & & \\
\hline & & & Izquierda & $\begin{array}{c}\text { Centro } \\
\text { izquierda }\end{array}$ & Centro & & $\begin{array}{l}\text { ntro } \\
\text { echa }\end{array}$ & Derecha & TOTAL \\
\hline No diálogo ++ & & & $30,9 \%$ & $37,4 \%$ & $52,3 \%$ & &, $7 \%$ & $71,9 \%$ & $48,4 \%$ \\
\hline & & & $-8,3^{*}$ & $-10,9 *$ & $4,0^{*}$ & &, $1 *$ & $7,4^{*}$ & - \\
\hline No diálogo + & & & $7,3 \%$ & $11,1 \%$ & $10,5 \%$ & & $6 \%$ & $7,7 \%$ & $10,1 \%$ \\
\hline & & & $-2,2 *$ & 1,6 & 0,7 & & 0,5 & $-1,3$ & - \\
\hline Equidistante & & & $11,3 \%$ & $10,3 \%$ & $11,8 \%$ & & $4 \%$ & $7,2 \%$ & $10,5 \%$ \\
\hline & & & 0,6 & $-0,3$ & $2,3^{*}$ & & $2 *$ & $-1,7$ & - \\
\hline Diálogo + & & & $7,7 \%$ & $12,9 \%$ & $10,4 \%$ & & $8 \%$ & $5,1 \%$ & $10,2 \%$ \\
\hline & & & $-2,0^{*}$ & $4,3^{*}$ & 0,2 & & $6^{*}$ & $-2,7^{*}$ & - \\
\hline Diálogo ++ & & & $42,8 \%$ & $28,4 \%$ & $15,0 \%$ & & $6 \%$ & $8,1 \%$ & $20,8 \%$ \\
\hline & & & $12,8^{*}$ & $9,2^{*}$ & $-7,4^{*}$ & & $6^{*}$ & $-4,9^{*}$ & - \\
\hline TOTAL & & & $100 \%$ & $100 \%$ & $100 \%$ & & $0 \%$ & $100 \%$ & $100 \%$ \\
\hline $\mathrm{RTC}> \pm 1,96(*) \mathrm{X}^{2}=$ & 8,9349 & $138 \mathrm{Gl}$ & $=16 \mathrm{Sig} ., 0 \mathrm{C}$ & & & & & & \\
\hline & & & PSOE & & & & & & \\
\hline & & 2008 & 2011 & 2008 & 2011 & 2008 & 2011 & 2008 & 2011 \\
\hline Policía / No diálogo & $\%$ & $27,0 \%$ & $40,3 \%$ & $64,5 \%$ & $65,3 \%$ & $54,5 \%$ & $41,8 \%$ & $41,0 \%$ & $49,3 \%$ \\
\hline ++ & RTC & $-22,4^{*}$ & $-7,0^{*}$ & $22,2^{*}$ & $17,0^{*}$ & 1,3 & $-10,4 *$ & - & - \\
\hline Policía / No diálogo & $\%$ & $9,0 \%$ & $10,6 \%$ & $12,9 \%$ & $10,1 \%$ & $9,1 \%$ & $8,9 \%$ & $10,4 \%$ & $9,7 \%$ \\
\hline+ & RTC & $-3,7^{*}$ & 1,2 & $3,8^{*}$ & 0,7 & $-0,2$ & $-1,7$ & - & - \\
\hline Equidistante & $\%$ & $18,0 \%$ & $11,9 \%$ & $10,6 \%$ & $8,6 \%$ & $13,6 \%$ & $11,6 \%$ & $15,2 \%$ & $10,6 \%$ \\
\hline & RTC & $6,0^{*}$ & 1,5 & $-6,0^{*}$ & $-3,5^{*}$ & $-0,2$ & $2,0 *$ & - & - \\
\hline Diálogo + & $\%$ & $15,3 \%$ & $12,5 \%$ & $4,8 \%$ & $6,8 \%$ & $9,1 \%$ & $10,8 \%$ & $11,4 \%$ & $9,8 \%$ \\
\hline & RTC & $9,6^{*}$ & $3,4^{*}$ & $-9,6^{*}$ & $-5,4^{*}$ & $-0,3$ & $2,3^{*}$ & - & - \\
\hline Diálogo ++ & $\%$ & $30,8 \%$ & $24,8 \%$ & $7,2 \%$ & $9,2 \%$ & $13,6 \%$ & $26,9 \%$ & $21,9 \%$ & $20,5 \%$ \\
\hline & RTC & $16,7^{*}$ & $4,1^{*}$ & $-16,6^{*}$ & $-15,0^{*}$ & $-0,9$ & $10,8^{*}$ & - & - \\
\hline TOTAL & & $100 \%$ & $100 \%$ & $100 \%$ & $100 \%$ & $100 \%$ & $100 \%$ & $100 \%$ & $100 \%$ \\
\hline
\end{tabular}


Tabla A7. Tolerancia / democracia, ideología y recuerdo de voto (2008 y 2011)

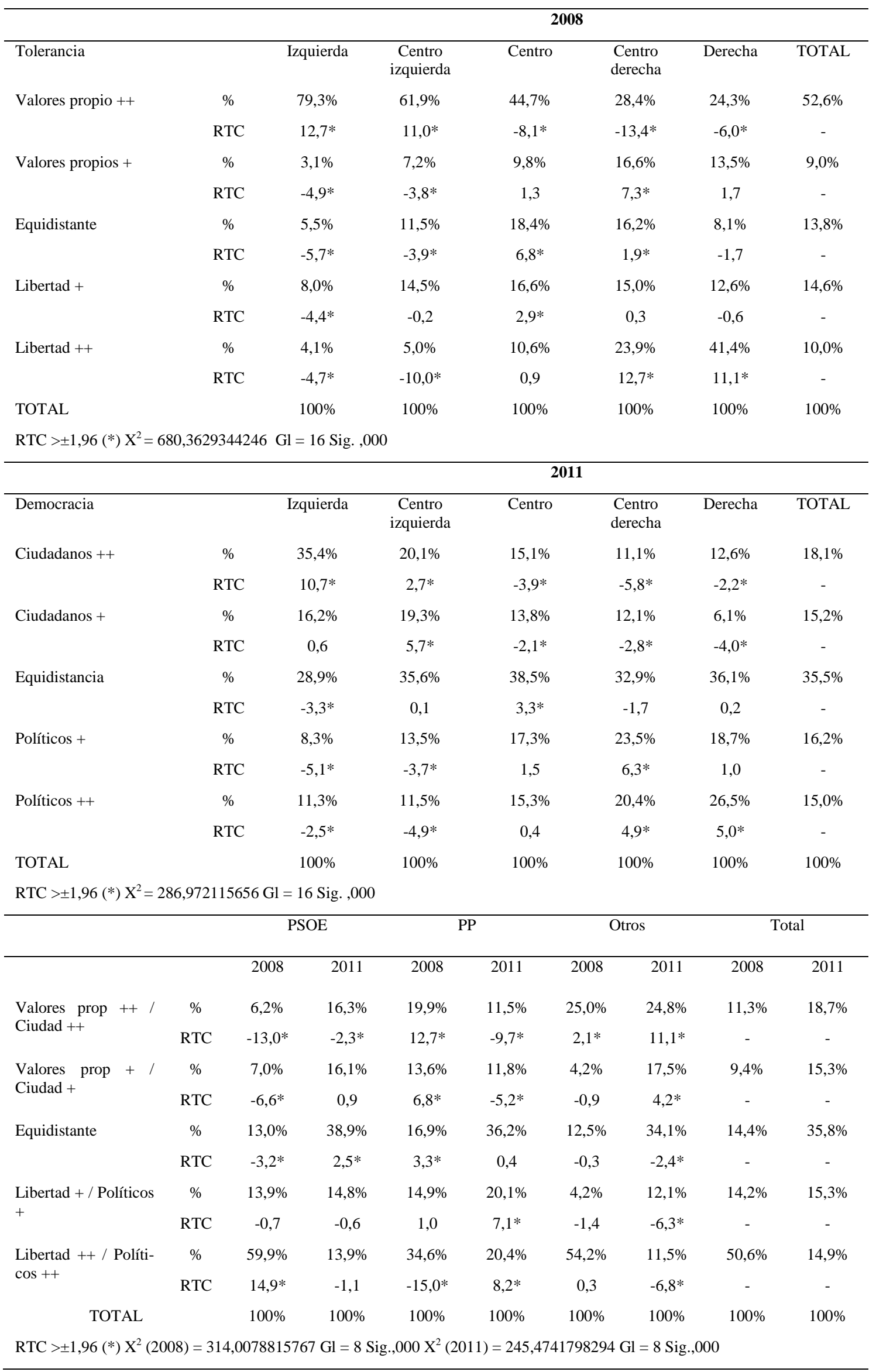


ANTÓN CASTROMIL. Antón R. Castromil es profesor del Departamento de Sociología VI de la Universidad Complutense de Madrid. Entre sus líneas de investigación se encuentran los efectos de los medios de comunicación en el comportamiento político de los ciudadanos, el negativismo mediático y polarización, así como comportamiento electoral, partidos políticos y teoría de la democracia.

PALMIRA CHAVERO. Profesora e investigadora del programa de Estudios Internacionales y Comunicación en FLACSO (Ecuador). Doctora en Ciencias de la Comunicación y Sociología por la Universidad Complutense de Madrid (España); Licenciada en Periodismo por la misma universidad. Investigadora del equipo de investigación Agenda y Voto, del Grupo de Investigación en Gobierno, Administración y Políticas Públicas (Gigapp); de proyectos de investigación internacionales (Journalistic Role Performance around the world; Worlds of Journalism Study; Culturas Periodísticas de Ecuador). Ha trabajado en la Universidad Complutense de Madrid (España), el Instituto Universitario de Investigación Ortega y Gasset y ha sido coordinadora del Laboratorio de Comunicación y Derechos (Instituto de Altos Estudios Nacionales, Ecuador). En 2011 recibió el "I Premio Jóvenes Investigadores Joan Prats", concedido por Gigapp y el Instituto Universitario de Investigación Ortega y Gasset. Coautora de una decena de libros, de artículos en revistas especializadas y de numerosas ponencias en congresos internacionales. Ha trabajado como periodista durante más de diez años; en 2008 recibió el "Premio Periodismo 8 de marzo".

Recibido: 10/09/2017

Aceptado: 21/10/2017 